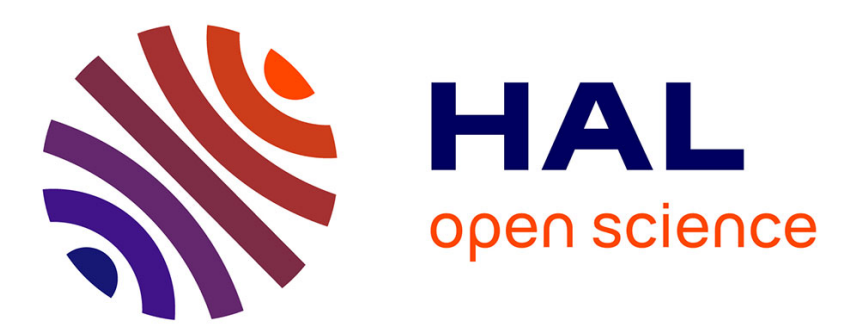

\title{
Broadband modeling of passive components via augmented equivalent circuit models
}

Sanda Lefteriu, Stefano Grivet-Talocia

\section{To cite this version:}

Sanda Lefteriu, Stefano Grivet-Talocia. Broadband modeling of passive components via augmented equivalent circuit models. 2018 IEEE MTT-S International Conference on Numerical Electromagnetic and Multiphysics Modeling and Optimization (NEMO), Aug 2018, Reykjavik, Iceland. hal-01897432

\section{HAL Id: hal-01897432 \\ https://hal.science/hal-01897432}

Submitted on 18 Oct 2018

HAL is a multi-disciplinary open access archive for the deposit and dissemination of scientific research documents, whether they are published or not. The documents may come from teaching and research institutions in France or abroad, or from public or private research centers.
L'archive ouverte pluridisciplinaire HAL, est destinée au dépôt et à la diffusion de documents scientifiques de niveau recherche, publiés ou non, émanant des établissements d'enseignement et de recherche français ou étrangers, des laboratoires publics ou privés. 


\section{Broadband modeling of passive components via augmented equivalent circuit models}

\author{
Sanda Lefteriu \\ Unité de Recherche Informatique Automatique \\ IMT Lille Douai, Univ. Lille, France \\ sanda.lefteriu@imt-lille-douai.fr
}

\author{
Stefano Grivet-Talocia \\ Dept. of Electronics and Telecommunications \\ Politecnico di Torino, Italy \\ stefano.grivet@polito.it
}

\begin{abstract}
This paper presents a gray-box modeling technique for obtaining stable and passive circuit models of electrical components. Starting from frequency response measurements and from an initial basic equivalent circuit model, a fully automated procedure performs series and parallel augmentations, the value of the added components being estimated by minimizing the deviation of the model response from measurements. An iterative application of this procedure yields accurate fits, allowing a compact representation of parasitic and second-order effects through suitably and automatically connected circuit elements.
\end{abstract}

\section{INTRODUCTION}

The continuous increase in the operating frequencies of integrated circuits requires accurate component models for inclusion in simulators during the design and verification phases. The fundamental (functional) behavior of basic components can be represented by a single element (an inductance $L$ or a capacitance $C$ or a resistance $R$ ), which, however, cannot capture parasitics and second-order effects and can only match the response of the hardware component over a limited frequency band. Instead, equivalent circuit models (ECMs) for spiral inductors [1], [2], ceramic capacitors [3] or thin-film resistors [4] must take into account parasitics, including skin and proximity effects, that are relevant at high frequencies.

There are two main approaches for ECM extraction. Starting from frequency responses obtained by direct measurement or electromagnetic simulation, the first approach fits a rational model to the measured transfer function [5], [6], including passivity enforcement as a post-processing step [7]. This approach is purely black-box: although a circuit synthesis is always possible, the obtained ECM does not reveal any physical insight. The other approach starts from an initial basic circuit model (which is preserved) and enriches it by adding components that aim at reproducing high-order effects and parasitics. The present paper focuses on this second approach.

Previous approaches for automated broadband model generation did not ensure stability and passivity by construction. In [8], the error between the measurements and the response

This work was supported by the ACRI foundation through the Young Investigator Training Program and the TD COST Action TD1307 European Model Reduction Network (EU-MORNET) as a Short Term Scientific Mission. Moreover, this work was completed in the framework of CE2I project (Convertisseur d'Energie Intgr Intelligent). CE2I is co-financed by European Union with the financial support of European Regional Development Fund (ERDF), French State and the French Region of Hauts-de-France. of the nominal circuit is modeled by a small number of pole-zero elements, the final model being found by blackbox fitting. Stability is enforced by pole-flipping and passivity is enforced a-posteriori. The same nonlinear optimization problem that we propose in this paper (see also [9]) is solved in [10] by expressing the unknown as a rational function, with its numerator and denominator coefficients found via least squares, stability being enforced by constraining positive coefficients. Passivity is then enforced via perturbation of the imaginary eigenvalues of a Hamiltonian matrix pencil [7]. These two approaches still make use of black-box fitting, although applied to incrementally update an initial basic ECM.

Our paper makes a step towards a fully-automated circuit generation for components described by frequency domain measurements, by avoiding black-box fitting. Starting from a nominal circuit (an incomplete ECM of a given component or subsystem), we propose to generate all possible augmentations of this circuit by inserting passive elements in series or in parallel to the existing ones. We treat each added circuit element independently, and we solve a nonlinear optimization problem via a Sanathanan-Koerner iteration combined with an alternating least-squares to find the element values that provide the best match to the given measurements. This process guarantees stability and passivity of the final model by construction, in addition to providing an automated placement of circuit elements required for parasitic representation.

The paper is structured as follows. Section II presents a review of the modified nodal analysis (MNA) used to describe electrical circuits. In Sec. III, we propose to perform all possible series and parallel augmentations of the nominal circuit. Section IV discusses the proposed approach for topological fitting. Lastly, Sec. V presents a numerical example validating our approach and Sec. VI concludes the paper.

\section{Circuit MODELING VIA MNA - SHORT REVIEW}

MNA [11], [12] is a powerful technique for formulating and solving circuit equations. The reduced incidence matrix A describes the topology of the circuit: its rows correspond to the nodes; each column, corresponding to a single element, is all-zero except for a single 1 and -1 pair at the two rows pointing to the nodes to which this element is connected (the row corresponding to a global reference node is removed). Kirchhoff's current and voltage laws describe the topological 
equations: $\mathbf{A i}_{b}=\mathbf{0}$ and $\mathbf{v}_{b}=\mathbf{A}^{T} \mathbf{e}$, where $\mathbf{i}_{b}$ are branch currents, $\mathbf{v}_{b}$, the branch voltages, and $\mathbf{e}$, the nodal voltages.

For the four types of elements: shunt dynamic (capacitors), shunt resistive (conductances), series dynamic (inductances) and series resistive (resistors), the columns of the incidence matrix are partitioned accordingly, as $\mathbf{A}_{C}, \mathbf{A}_{G}, \mathbf{A}_{L}, \mathbf{A}_{R}$, respectively. We also define corresponding diagonal matrices $\mathbf{C}_{b}, \mathbf{G}_{b}, \mathbf{L}_{b}, \mathbf{R}_{b}$ storing the values of the elements (including, as a particular case, empty matrices if no elements of a given class are present). The constitutive equations for voltage controlled (shunt) elements are $\mathbf{i}_{G}=\mathbf{G}_{b} \mathbf{v}_{G}$ and $\mathbf{i}_{C}=s \mathbf{C}_{b} \mathbf{v}_{C}$, while for current controlled (series) elements, they are $\mathbf{v}_{R}=$ $\mathbf{R}_{b} \mathbf{i}_{R}$ and $\mathbf{v}_{L}=s \mathbf{L}_{b} \mathbf{i}_{L}$, where $\mathbf{v}_{\nu}, \mathbf{i}_{\nu}$ collect branch voltages and currents on branches of type $\nu=\{C, G, L, R\}$.

The unknowns of the MNA system are the node voltages and the branch currents for the series elements: $\mathbf{x}=$ $\left[\begin{array}{lll}\mathbf{e} & \mathbf{i}_{L} & \mathbf{i}_{R}\end{array}\right]^{T}$, yielding a descriptor-form representation

$$
\mathbf{E}_{d a e} \dot{\mathbf{x}}(t)=\mathbf{A}_{d a e} \mathbf{x}(t)+\mathbf{B u}(t), \quad \mathbf{y}(t)=\mathbf{C x}(t),
$$

with transfer matrix $\mathbf{H}(s)=\mathbf{C}\left(s \mathbf{E}_{d a e}-\mathbf{A}_{d a e}\right)^{-1} \mathbf{B}$, where $\mathbf{B}$ and $\mathbf{C}$ are input and output mappings that depend on the adopted input-output representation $(Z, Y$, or $S)$, and

$$
\mathbf{E}_{d a e}=\left[\begin{array}{ccc}
\mathbf{A}_{C} \mathbf{C}_{b} \mathbf{A}_{C}^{T} & \mathbf{0} & \mathbf{0} \\
\mathbf{0} & \mathbf{L}_{b} & \mathbf{0} \\
\mathbf{0} & \mathbf{0} & \mathbf{0}
\end{array}\right], \mathbf{A}_{d a e}=-\left[\begin{array}{ccc}
\mathbf{A}_{G} \mathbf{G}_{b} \mathbf{A}_{G}^{T} & \mathbf{A}_{L} & \mathbf{A}_{R} \\
-\mathbf{A}_{L}^{T} & \mathbf{0} & \mathbf{0} \\
-\mathbf{A}_{R}^{T} & \mathbf{0} & \mathbf{R}_{b}
\end{array}\right] .
$$

\section{Circuit augmentation}

Given a nominal circuit, augmentations are performed:

1) in parallel with shunt $C$ and $G$ elements between any two nodes, if no such elements exist. New columns are appended to the incidence submatrices $\mathbf{A}_{C}, \mathbf{A}_{G}$, and the (unknown) element value is added to matrices $\mathbf{C}_{b}, \mathbf{G}_{b}$.

2 ) in series with $L$ and $R$ elements between any two nodes, if no such elements exist. The branch is split into seriesconnected branches by adding new nodes. The row size of the incidence submatrices $\mathbf{A}_{L}$ and $\mathbf{A}_{R}$ grows by two, some entries are modified to account for the split, while a new column is appended to account for the new element. Consistently, $\mathbf{L}_{b}$ and $\mathbf{R}_{b}$ are also augmented.

To minimize user interaction, we generate all possible series and parallel augmentations of a nominal (starting) circuit according to these rules. Initial component values are set to 0 .

\section{ELEMENT PERTURBATION}

The next step is to identify appropriate values for the newly added elements. In practice, all elements (including the preexisting ones) should be re-optimized due to the modified topology. Our approach is to perturb each element value independently to minimize the error between the response of the model and the given measurements, looping through all elements via an alternating least squares iteration.

\section{A. Single element perturbation}

We distinguish between the two different element types

- shunt element: if connected between nodes $k$ and $l$,we define an all vanishing selector vector $\xi$ with the same size as the MNA matrices $\mathbf{E}_{d a e}, \mathbf{A}_{d a e}$, except entries $\xi_{k}=1$ and $\xi_{l}=-1$.

- series element: we define $\xi=\left[\begin{array}{lll}\mathbf{0}_{n} & \mathbf{e}_{L} & \mathbf{0}_{n_{R}}\end{array}\right]^{T}$ for an inductance and $\xi=\left[\begin{array}{lll}\mathbf{0}_{n} & \mathbf{0}_{n_{L}} & \mathbf{e}_{R}\end{array}\right]^{T}$ for a resistance, with $\mathbf{0}_{\nu}$ being a null vector of size $\nu=\left\{n, n_{R}, n_{L}\right\}$ (number of nodes except reference, of resistors, of inductors, respectively), and where $\mathbf{e}_{L}$ is the unit vector with a single 1 at the position of the corresponding inductor, and similarly for $\mathbf{e}_{R}$.

We define $\mathcal{G}:=s \mathbf{E}_{d a e}-\mathbf{A}_{d a e}$. A change in the element value induces a perturbation $\mathcal{G}=\mathcal{G}_{0}+\Delta \alpha \xi \xi^{T}$, where $\mathcal{G}_{0}$ refers to the nominal circuit, and $\Delta \alpha$ is the perturbation in the value of the element. For a dynamic element, $\Delta \alpha=s \Delta C_{i}$ or $\Delta \alpha=s \Delta L_{i}$. For static elements, $\Delta \alpha=\Delta G_{i}$ or $\Delta \alpha=\Delta R_{i}$. The ShermanMorrison formula yields

$$
\mathcal{G}^{-1}=\mathcal{G}_{0}^{-1}-\frac{\mathcal{G}_{0}^{-1} \Delta \alpha \xi \xi^{T} \mathcal{G}_{0}^{-1}}{1+\Delta \alpha \xi^{T} \mathcal{G}_{0}^{-1} \xi} .
$$

Hence, the transfer function is

$$
\mathbf{H}(s)=\mathbf{C} \mathcal{G}^{-1} \mathbf{B}=\mathbf{H}_{0}(s)-\Delta \alpha \frac{\mathbf{F}(s)}{1+\Delta \alpha K(s)} .
$$

where $\mathbf{F}(s)=\mathbf{C G}_{0}^{-1} \xi \xi^{T} \mathcal{G}_{0}^{-1} \mathbf{B}, K(s)=\xi^{T} \mathcal{G}_{0}^{-1} \xi$, and $\mathbf{H}_{0}(s)=\mathbf{C G}_{0}^{-1} \mathbf{B}$. We now define the error between the measurements $\mathbf{H}_{i}$ and the response of the perturbed system at frequencies $\omega_{i}$ as

$$
\mathcal{E}=\sum_{i=1}^{N}\left\|\mathbf{H}\left(\mathrm{j} \omega_{i}\right)-\mathbf{H}_{i}\right\|_{F}^{2}=\sum_{i=1}^{N}\left\|\Delta \mathbf{H}_{i}-\mathbf{F}_{i} \frac{\Delta \alpha}{1+\Delta \alpha \cdot K_{i}}\right\|_{F}^{2},
$$

with $\Delta \mathbf{H}_{i}=\mathbf{H}_{0}\left(\mathrm{j} \omega_{i}\right)-\mathbf{H}_{i}, \mathbf{F}\left(\mathrm{j} \omega_{i}\right)=\mathbf{F}_{i}$ and $K\left(\mathrm{j} \omega_{i}\right)=K_{i}$. Naturally, we wish to minimize this error with respect to $\Delta \alpha$. The optimization parameter $\Delta \alpha$ appears in the numerator and denominator, making this problem nonlinear. With the Sanathanan-Koerner (SK) iteration [13], we relax the problem by using $\Delta \alpha_{k-1}$ from the previous iteration in the denominator, yielding a linear problem for the current $\Delta \alpha_{k}$

$$
\min _{\Delta \alpha_{k}} \sum_{i=1}^{N}\left\|\frac{\Delta \mathbf{H}_{i}+\left(\Delta \mathbf{H}_{i} K_{i}-\mathbf{F}_{i}\right) \Delta \alpha_{k}}{1+\Delta \alpha_{k-1} \cdot K_{i}}\right\|_{F}^{2}
$$

which is solved via linear least-squares.

\section{B. Several elements}

For perturbing several elements, we employ alternating least squares, by optimizing each element independently using Sec. IV-A. This iterative process is run until convergence or until a maximum number of iterations is reached. The identification is effective if the number of raw samples is much larger than the number of elements that are required for an accurate fit. 

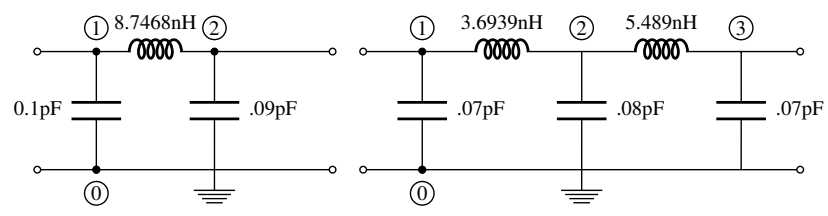

Fig. 1. $\pi$ model in low frequency

Fig. 2. Broadband $2 \pi$ model

\section{NUMERICAL EXAMPLES}

We illustrate the proposed approach on a 2-port spiral inductor test case, initially characterized by 200 S-parameter samples from $100 \mathrm{MHz}$ up to $10 \mathrm{GHz}$ obtained from a fullwave electromagnetic simulation. The initial nominal circuit is a single inductance $L_{\text {nom }}$ connected between the two ports, both referenced to a common ground node, whose value is also to be identified. We assume that this inductance is representative only at low frequency. Therefore, we refine the ECM as follows.

1) An initial ECM with two shunt capacitors (Fig. 1) is identified using data only up to $5 \mathrm{GHz}$;

2) A second nominal ECM is generated by halving the component values and cascading two identical sections (Fig. 2) to better reproduce proximity effects [1], [2]. These values are then optimized to match the full frequency behavior.

3) The automated augmentation from Sec III is run on the above ECM without additional user intervention.

The resulting circuit is shown in Fig. 3 (some elements have small values and can be removed without loss of accuracy, however, to illustrate the automated augmentation, we show them nevertheless). Responses for this ECM match the field solver data closely on the whole frequency band (Fig. 4). Similar performance was observed on other examples (not shown due to space limitations).

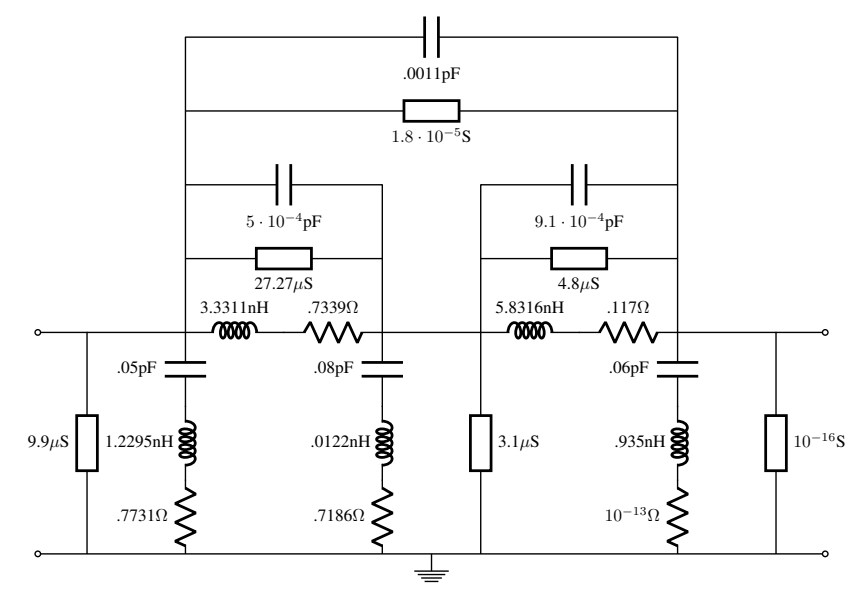

Fig. 3. Circuit obtained after optimizing the element values for the circuit obtained by all possible series and parallel augmentations of the $2 \pi$ configuration in Fig. 2
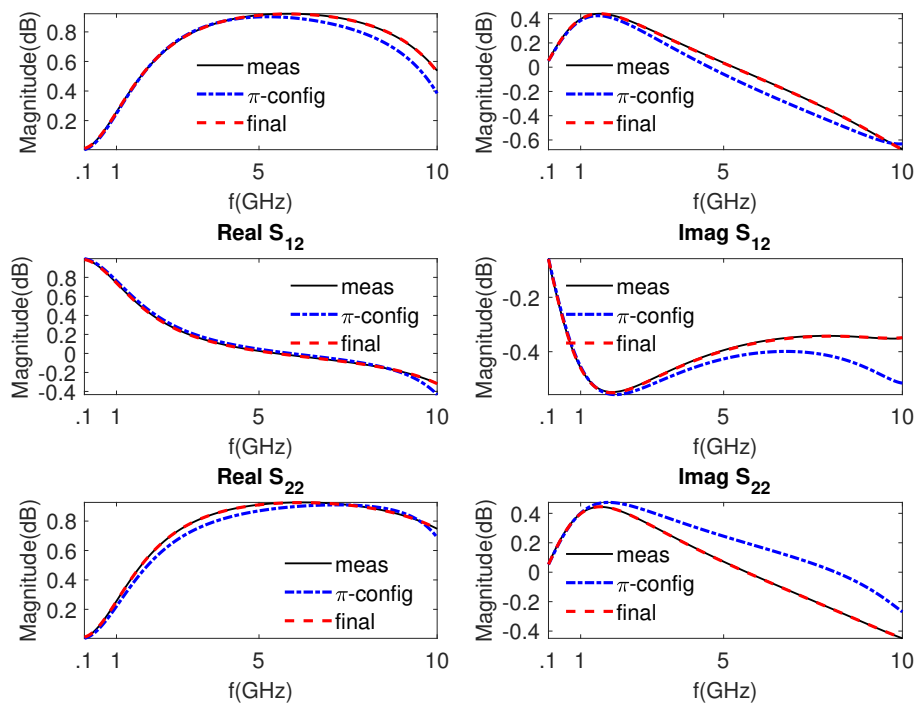

Fig. 4. Fitting the augmented circuit on the entire frequency band; measurements are shown together with the models from Fig. 1 and Fig. 3

\section{CONCLUSION}

This paper aims to make a step towards automated generation of equivalent circuit models for components. These models are valid in a broad frequency band and are stable and passive by construction. We observed that the scheme works quite well on basic components such as spiral inductors and capacitors, leading to simple topologies with few elements.

The algorithm may run into difficulties if the size of the ECM grows too large, or if the topology of the nominal circuit is not appropriate for the application at hand. In the future, we will consider combining the SK process with a Newton iteration and use regularization for alternate least-squares, to improve and speed up convergence.

\section{REFERENCES}

[1] Y. Cao, R. A. Groves, X. Huang, N. D. Zamdmer, J. O. Plouchart, R. A. Wachnik, T.-J. King, and C. Hu, "Frequency-independent equivalentcircuit model for on-chip spiral inductors," IEEE J. Solid-State Circuits, vol. 38, no. 3, pp. 419-426, Mar 2003.

[2] X. Wang, J. Ren, F. Yang, W. Zheng, Q. Yu, N. Ning, and M. Yang, "A wide-band $2 \pi$ equivalent-circuit model for spiral inductors on silicon," in International Conference on Communications, Circuits and Systems, vol. 4, June 2006, pp. 2605-2609.

[3] C. R. Sullivan, Y. Sun, and A. M. Kern, "Improved distributed model for capacitors in high-performance packages," in 2002 IEEE Industry Applications Conference, vol. 2, Oct 2002, pp. 969-976.

[4] Y. Li and Z. Wei, "A high-frequency scalable model for thin-film resistor on gan substrate," in International Conference on Communication Technology (ICCT), Oct 2015, pp. 589-592.

[5] B. Gustavsen and A. Semlyen, "Rational approximation of frequency domain responses by vector fitting," IEEE Trans. Power Del., vol. 14, pp. 1052-1061, Jul. 1999.

[6] S. Lefteriu and A. C. Antoulas, "A new approach to modeling multiport systems from frequency domain data," IEEE Trans. Comput.-Aided Design Integr. Circuits Syst., vol. 29, no. 1, pp. 14 -27, Jan. 2010.

[7] S. Grivet-Talocia and B. Gustavsen, Passive Macromodeling: Theory and Applications. New York: John Wiley and Sons, 2015.

[8] J. Kolstad, C. Blevins, J. M. Dunn, and A. Weisshaar, "A new circuit augmentation method for modeling of interconnects and passive components," IEEE Trans. Adv. Packag., vol. 29, no. 1, pp. 67-77, Feb 2006. 
[9] S. Lefteriu and S. Grivet-Talocia, "Topological fitting: broadband modeling of passive components via augmented equivalent circuit models," IFAC-PapersOnLine, vol. 51, no. 2, pp. 451 - 456, 2018, 9th Vienna International Conference on Mathematical Modelling.

[10] D. Paul, M. S. Nakhla, R. Achar, and A. Weisshaar, "Broadband modeling of high-frequency microwave devices," IEEE Trans. Microw. Theory Tech., vol. 57, no. 2, pp. 361-373, Feb 2009.

[11] C. W. Ho, A. Ruehli, and P. Brennan, "The modified nodal approach to network analysis," IEEE Trans. Circuits Syst., vol. 22, no. 6, pp. 504-509, 1975.

[12] J. Vlach and K. Singhal, Computer methods for circuit analysis and design. Springer, 1983.

[13] C. Sanathanan and J. Koerner, "Transfer function synthesis as a ratio of two complex polynomials," IEEE Trans. Autom. Control, vol. 8, no. 1, pp. 56-58, jan 1963. 